\title{
Projeções de Custos e Rentabilidade do Setor Sucroenergético na Região Nordeste para a Safra 2015/16
}

\section{Costs projections and Sugarcane Industry Profitability in the Northeast for the 2015/16 season}

\author{
Luiz Fernando Sansigolo Xavier ${ }^{1 *}$; Aline Bigaton²; André Felipe Danelon; \\ Haroldo José Torres da Silva ${ }^{4}$, Pedro Valentim Marques ${ }^{5}$
}

\footnotetext{
${ }^{1}$ ESALQ/USP - Graduando em Ciências Econômicas - Rua Alexandre Herculano 120, sala T4, Vila Monteiro - CEP 13418-445 - Piracicaba (SP), Brasil

2 UNICAMP - Mestranda em Planejamento de Sistemas Energéticos (Faculdade de Engenharia Mecânica) - Rua Alexandre Herculano 120, sala T4, Vila Monteiro - CEP 13418-445 - Piracicaba (SP), Brasil

${ }^{3}$ ESALQ/USP- Graduando em Ciências Econômicas - Rua Alexandre Herculano 120, sala T4, Vila Monteiro - CEP 13418-445 - Piracicaba (SP), Brasil

4 ESALQ/USP - Mestrando em Economia Aplicada (Departamento de Economia, Administração e Sociologia) - Rua Alexandre Herculano 120, sala T4, Vila Monteiro - CEP 13418-445 - Piracicaba (SP), Brasil

${ }^{5}$ ESALQ/USP - Professor Titular (Departamento de Economia, Administração e Sociologia) - Av. Pádua Dias 11 - CEP 13418-900 - Piracicaba (SP), Brasil
}

\section{Resumo}

O presente artigo tem por finalidade analisar o desempenho do setor sucroenergético na região nordeste, utilizando-se da estrutura e ferramental teórico desenvolvido ao longo das oito safras nas quais o Programa de Educação Continuada em Economia e Gestão de Empresas PECEGE atuou com levantamento de custos, até o presente momento. Durante o período analisado observou-se variação de custos e eficiência, com modificações nos preços dos produtos em termos reais e nas margens econômicas das empresas. Além da evolução, desde a safra $07 / 08$, são elaborados cenários e projeções com o objetivo de entender o comportamento do setor nas próximas safras na região. A partir deles, nota-se que componentes macroeconômicos desfavoráveis trazem dificuldades à administração das usinas, que precisam se mostrar mais eficientes para superar as dificuldades apontadas para as próximas safras.

Palavras-chave: cana-de-açúcar, produção, agroindustrial

\section{Abstract}

This article aims to analyze the performance of the sugarcane industry in the Northeast, using the structure and theoretical tools developed over the eight seasons in which the Programa de Educação Continuada em Economia e Gestão de Empresas - PECEGE served with lifting costs to date. During the analyzed period, there was variation in costs and effectiveness, changes in product prices and the economic margins of the companies. In addition to the evolution, since the 07/08 season, they are designed scenarios and projections in order to understand the behavior of the sector in the coming seasons in the region. From them, it is noted that unfavorable macroeconomic components bring difficulties to the administration of the plants that need to show more efficiency to overcome the difficulties identified for the next season.

Keywords: sugarcane, production, agro-industrial

\footnotetext{
1 *Autor correspondente <luizsansigolo@pecege.com> Enviado: 29 jan. 2016

Aprovado: 30 mar. 2016
} 


\section{Introdução}

Desde a safra 2007/2008 o Programa de Educação Continuada em Economia e Gestão de Empresas - PECEGE da Escola Superior de Agricultura "Luiz de Queiroz" ESALQ/USP, juntamente com a Confederação Nacional da Agricultura e Pecuária CNA, realiza anualmente o Levantamento de Custos de Produção de Cana-de-Açúcar, Açúcar, Etanol e Bioeletricidade nas principais regiões produtoras do Brasil. Tal pesquisa forma um importante arcabouço que permite entender a evolução trazendo uma análise mais detalhada do setor.

No período compreendido entre as safras 2007/08 e 2014/15, fazendo-se uma análise específica para a região Nordeste, a produtividade agrícola sofreu forte oscilação, com quedas expressivas seguidas de períodos de recuperação. Após duas safras consecutivas de elevação, a produtividade atual mostra recuperação, chegando ao patamar de $60 \mathrm{t} \mathrm{ha}^{-1}$, porém, permanece abaixo da média histórica. Apesar dos gastos com tratos culturais de cana soca apresentarem tendência média de crescimento de $3,5 \%$ ao ano, a qualidade da matéria prima na safra atual é $2,2 \%$ menor que na safra 2007/2008. Medida pelo teor de açúcares totais recuperáveis - ATR, a qualidade da

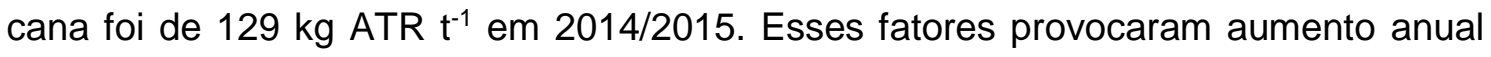
médio de $1,5 \%$ nos custos de produção no período.

Pela ótica industrial, na safra 2014/15, os custos com manutenção na indústria foram $22 \%$ menores do que os observados em 2007/08. No entanto, os custos com mão de obra apresentaram aumento real de $6 \%$ a.a. durante o período.

O aumento dos custos de produção, puxado pela deterioração dos canaviais e pela elevação da mão de obra, foi superior ao aumento real dos preços dos produtos e tem pressionado a margem econômica das usinas e dos produtores de cana-de-açúcar. Com menor rentabilidade, as necessidades de novos investimentos para reverter a baixa eficiência foram comprometidas. Além disso, a redução do preço do açúcar no mercado internacional e as alterações no mercado doméstico do etanol levaram a alterações no mix de produção das usinas.

Os custos de produção dos principais produtos do setor ${ }^{2}$ aumentaram entre $16 \%$ e $18 \%$ no período. Quanto à rentabilidade, os dois tipos de açúcar obtiveram margens positivas na maior parte das safras analisadas, ao passo que o etanol raramente alcançou rentabilidade.

A maior rentabilidade do açúcar na região Nordeste explica o mix açucareiro por volta de $60 \%$ no período. A Tabela 1 apresenta a proporção de cana destinada à

\footnotetext{
2 Os principais produtos do setor são: açúcar branco, açúcar VHP (Very High Polarization), etanol anidro e etanol hidratado.
} 
fabricação de açúcar e etanol ao longo das safras contempladas pelo levantamento de custos de produção do PECEGE/CNA.

Tabela 1. Evolução do mix de produção das usinas da região Nordeste

\begin{tabular}{|c|c|c|c|c|c|c|c|c|}
\hline \multirow{2}{*}{ Mix Produção } & \multicolumn{7}{|c|}{$\%$ na safra } & \multirow[b]{2}{*}{$2014 / 15$} \\
\hline & $2007 / 08$ & 2008/09 & $2009 / 10$ & $2010 / 11$ & $2011 / 12$ & $2012 / 13$ & $2013 / 14$ & \\
\hline Açúcar & 68,00 & 64,90 & 73,00 & 64,98 & 60,40 & 67,12 & 68,47 & 65,85 \\
\hline Branco & 43,50 & 55,00 & 63,54 & 51,01 & 41,43 & 32,77 & 52,37 & 30,40 \\
\hline VHP & 56,50 & 45,00 & 36,46 & 35,35 & 58,58 & 67,23 & 47,63 & 69,60 \\
\hline Etanol & 32,00 & 35,10 & 27,00 & 35,02 & 39,60 & 32,88 & 31,53 & 34,15 \\
\hline Anidro & 73,00 & 58,00 & 50,94 & 45,82 & 38,04 & 64,78 & 76,06 & 77,86 \\
\hline Hidratado & 27,00 & 42,00 & 49,06 & 54,18 & 61,96 & 35,22 & 23,94 & 22,14 \\
\hline
\end{tabular}

Fonte: PECEGE/CNA, 2015

Como consequência da deterioração dos canaviais e perda de eficiência na indústria, o custo total de produção agroindustrial aumentou cerca de $15 \%$ ao longo do período. Com isso, a margem econômica das usinas passou de positiva entre as safras 2009/10 e 2011/12, para negativas a partir da safra 2013/14. A Tabela 2 ilustra essa evolução dos custos e margens econômicas.

Tabela 2. Custo total de produção de cana-de-açúcar (própria e de fornecedores), agroindustrial (em $\mathrm{R} \$$ /tonelada) e margem econômica

\begin{tabular}{lcccccccccc}
\hline & \multicolumn{7}{c}{ Custo da safra } & \\
\cline { 2 - 9 } Fatores de Produção & & $2007 / 08$ & $2008 / 09$ & $2009 / 10$ & $2010 / 11$ & $2011 / 12$ & $2012 / 13$ & $2013 / 14$ & $2014 / 15$ & $\begin{array}{c}\% \\
\text { anual }\end{array}$ \\
\hline Cana própria $\left(\mathrm{R} \$ \mathrm{t}^{-1}\right)$ & 79,11 & 86,52 & 80,48 & 83,85 & 88,74 & 109,24 & 97,10 & 96,01 & 1,50 \\
Cana fornecedor $\left(\mathrm{R} \$ \mathrm{t}^{-1}\right)$ & 54,32 & 67,59 & 83,29 & 93,44 & 84,14 & 82,73 & 73,93 & 70,53 & 1,10 \\
Custo total $\left(\mathrm{R} \$ \mathrm{t}^{-1}\right)$ & 112,48 & 120,55 & 123,42 & 126,47 & 126,29 & 143,08 & 134,50 & 129,34 & 1,00 \\
Margem $\mathrm{CT}^{3}$ & $-0,21$ & $-0,08$ & 0,14 & 0,03 & 0,16 & 0,00 & $-0,06$ & $-0,08$ & 3,36 \\
\hline
\end{tabular}

Fonte: PECEGE/CNA, 2015

As margens das usinas apresentam oscilação durante o período apresentado, sendo negativas nas últimas duas safras, com expectativa que essa tendência se mantenha para a safra atual. As projeções do cenário microeconômico das usinas associadas ao arrefecimento da economia brasileira emitem sinal de alerta para as usinas durante a safra 2015/16. Pelo lado positivo, a recuperação da produtividade dos canaviais tende a amenizar as expectativas de perdas para as próximas safras.

\footnotetext{
${ }^{3} \mathrm{~A}$ margem CT (custo total) refere-se à relação entre a receita obtida com a venda dos produtos produzidos e o custo total, dada pela fórmula: $\left(\frac{\text { receita total-custo total }}{\text { custo total }}\right) \times 100$.
} 
A partir da compreensão dos dados de safras passadas, torna-se possível fazer inferências sobre safras futuras, dando ao mercado e ao Estado informação para a tomada de decisão, se antecipando a eventuais problemas. Assim, o presente trabalho objetiva projetar os custos do setor canavieiro para a região Nordeste do Brasil na safra 2015/16, utilizando-se da análise do setor nos últimos anos em conjunto com as premissas necessárias para a projeção.

\section{Metodologia}

Modelo de previsão de custos e elaboração de cenários

As estimativas de custos e rentabilidades projetadas para a safra 2015/16 foram baseadas em: i) o modelo de cálculo de custos de produção do PECEGE/CNA para a região Nordeste; ii) banco de dados do fechamento da safra 2014/15 do PECEGE/CNA; iii) dados do CONSECANA-AL sobre fechamento da safra 2014/15 e projeção da safra 2015/16; iv) informações levantadas com usinas, sindicatos e associações no mês de agosto de $2015 \mathrm{e} \mathrm{v}$ ) pesquisa interna sobre indicadores relevantes para o setor, bem como análise do histórico dos Levantamentos de Custos do PECEGE/CNA. As variações das principais contas de custos coletadas pelo PECEGE em seus levantamentos foram estimadas a partir das variações dos índices de preços no período compreendido entre agosto de 2014 e julho de 2015. A Tabela 3 apresenta as variações de preços para cada item ajustado no modelo de custos, a magnitude do ajuste e a sua referência.

Os cenários otimista e pessimista foram definidos como variações positivas e negativas, respectivamente, de 5,00\% sobre o valor definido como base. As tabelas geradas avaliam as margens em relação ao Custo Total - CT ou ao Custo Operacional Total - COT $^{4}$.

\section{Premissas do modelo}

As premissas técnicas utilizadas nas projeções de custos para a safra 2015/16 foram: i) produtividade média do canavial de 60,0 $\mathrm{t} \mathrm{ha}^{-1}$; ii) qualidade de 131,05 kg ATR $\mathrm{t}^{-1}$ de cana de fornecedores e 130,0 kg ATR $\mathrm{t}^{-1}$ de cana própria; iii) preço do ATR de $\mathrm{R} \$$ $0,590 \mathrm{~kg}^{-1}$, ou seja, preço da cana de fornecedores de $\mathrm{R} \$ 77,32$ por tonelada; iv) ATR padrão de $114,09 \mathrm{kgATR}^{-1}$; v) preço de arrendamento de 14,96 $\mathrm{t} \mathrm{ha}^{-1}$; vi) custo CCT de

\footnotetext{
${ }^{4}$ Enquanto o CT representa a soma dos custos desembolsáveis acrescidos os custos de depreciação e remunerações da terra e do capital, o COT apresenta as margens em relação apenas ao custo operacional, isto é, o CT deduzindo-se as remunerações da terra e do capital.
} 
$\mathrm{R} \$ 33,75 \mathrm{t}^{-1}$; vii) perdas industriais comuns ${ }^{5}$ de $7,82 \%$; viii) diminuição da área colhida em $1 \%$ e ix) taxa de utilização de $87,9 \%$ da capacidade industrial. A capacidade de processamento de cana de uma unidade industrial adotada no modelo de custos foi atualizada passando de 1.200.000 t/safra para 1.500.000 t/safra, dado o aumento da unidade média produtiva na região nordeste.

Destacam-se na Tabela 3 as variações mais relevantes para projeção de custos na safra 2015/16, a saber, a) aumento de $8,6 \%$ do preço do ATR; b) aumento de 9,80\% dos salários industriais, administrativos e agrícolas; c) 9,20\% de aumento no preço do óleo diesel e d) $16,1 \%$ de aumento no custo de CCT. O teor de ATR por tonelada de cana-de-açúcar se manteve estável na projeção, com uma variação estimada em 1\%.

Tabela 3. Variações de preços consideradas para a projeção dos custos de produção agroindustriais para a safra 2015/2016: Nordeste

\begin{tabular}{|c|c|c|c|}
\hline Área & Insumo - Produto & $\begin{array}{c}\text { Variação considerada } \\
\text { na projeção de } \\
\text { custos } \\
\end{array}$ & $\begin{array}{c}\text { Fonte } \\
\text { considerada }\end{array}$ \\
\hline & & $\%$ & \\
\hline \multirow{8}{*}{ INDUSTRIAL } & Diesel & 9,20 & COPOM \\
\hline & Eletrodos & 6,21 & $|G P-D|^{1}$ \\
\hline & Energia Elétrica & 50,10 & COPOM \\
\hline & Insumos Químicos & 6,21 & IGP-DI \\
\hline & Lubrificantes & 5,02 & $\mathrm{IPA}^{2}$ \\
\hline & Sacaria - $50 \mathrm{~kg}$ & 6,21 & IGP-DI \\
\hline & Mão-de-Obra & 8,34 & Sindaçúcar-AL \\
\hline & $\begin{array}{l}\text { Peças e serviços de } \\
\text { manutenção }\end{array}$ & 6,21 & IGP-DI \\
\hline \multirow{7}{*}{ AGRÍCOLA } & Calcário & 5,02 & IPA \\
\hline & Fertilizantes & 15,00 & US\$3 \\
\hline & Herbicidas & 5,02 & IPA \\
\hline & Inseticidas & 5,02 & IPA \\
\hline & Mão-de-Obra & 9,80 & Sindaçúcar-AL \\
\hline & Mudas & 8,56 & ATR $^{4}$ \\
\hline & Peças e serviços de maquinário & 6,21 & IGP-DI \\
\hline ADMINISTRATIVC & Mão-de-Obra & 9,80 & Sindaçúcar-AL \\
\hline
\end{tabular}

Fonte: PECEGE/CNA, 2015; SINDAÇÚCAR-AL, 2015 e BACEN, 2015

1Variação observada do IGP-DI entre julho/2015 e junho/2016.

2Variação observada do IPA entre julho/2015 e junho/2016.

3 Variação calculada com base na variação do dólar e do barril de petróleo.

${ }^{4}$ Variação observada do ATR baseada nos preços médios estimados para a safra 2015/2016.

\footnotetext{
${ }^{5}$ Perdas industriais comuns referem-se ao agregado das perdas relacionadas ao processamento da cana, ou seja, perdas na lavagem, extração, na torta e indeterminada.
} 
Quanto aos preços dos produtos, projeta-se aumento de 9,20\% para o etanol hidratado, baseado na variação do preço do diesel apontada na $192^{\mathrm{a}}$ ata do COPOM ${ }^{6}$. Já para o etanol anidro, a variação de 9,4\% foi calculada levando-se em conta a paridade de preços com o etanol hidratado. Para o açúcar VHP, a expectativa de variação em $7,3 \%$ foi baseada nos preços médios dos contratos futuros do açúcar, com vencimento em maio de 2016 e do dólar com vencimento em julho de 2016. As datas dos vencimentos foram escolhidas tendo em vista o pico de produção da safra e o aumento médio do dólar esperado de uma safra para a outra. Para o açúcar branco (a exemplo do etanol anidro), o aumento de 7,6\% foi obtido através da paridade de preços entre o açúcar branco e o VHP nas últimas 3 safras.

\section{Resultados e Discussão}

As projeções de custos do setor sucroenergético para a safra 2015/16 na região Nordeste são apresentadas na Tabela 4. Todos são superiores ao custo médio de produção das últimas 3 safras na região. Enquanto o custo de produção de açúcar VHP deve ser $6 \%$ maior que a média, o açúcar branco deve ter aumento de $5 \%$. Os dois tipos de etanol devem ter seus custos de produção acrescidos em $4 \%$. O custo de produção da cana própria, por sua vez, deve apresentar crescimento real de $5 \%$. Mais uma safra em que a indústria sucroenergética deve destacar atenção para otimização de processos e absorver os impactos negativos da baixa rentabilidade dos negócios.

Tabela 4. Projeção dos custos de produção agroindustriais para a safra 2015/2016: Nordeste

\begin{tabular}{cccccc}
\hline Custo & Cana & $\begin{array}{r}\text { Accúcar } \\
\text { Branco }\end{array}$ & Açúcar VHP & Etanol Anidro & $\begin{array}{c}\text { Etanol } \\
\text { Hidratado }\end{array}$ \\
\hline & & ${\mathrm{R} \$ \mathrm{t}^{-1}}$ & & \multicolumn{3}{c}{$\mathrm{R} \$ \mathrm{~m}^{-3}$} \\
$\mathrm{COE}$ & 65,38 & 850,71 & 799,01 & 1345,39 & 1276,90 \\
$\mathrm{COT}$ & 90,23 & $1.012,39$ & 960,20 & $1.616,81$ & $1.534,50$ \\
$\mathrm{CT}$ & 106,06 & $1.168,22$ & $1.115,56$ & $1.878,41$ & $1.782,78$ \\
\hline
\end{tabular}

Fonte: PECEGE/CNA, 2015

A partir das projeções de custos de produção para a safra 2015/16, foram criados doze cenários, formados pela combinação de estimativas sobre a produtividade agrícola da cana e o preço do ATR ou dos produtos derivados da cana. As projeções de rentabilidades para a produção de cana-de-açúcar sobre o custo são negativas em

\footnotetext{
${ }^{6}$ Disponível em http://www.bcb.gov.br/?COPOM192
} 
todos os cenários, tanto para o COT como para o CT, conforme pode ser observado nas Tabelas 5 e 6.

Tabela 5 - Análise de sensibilidade para a rentabilidade agrícola (margem CT): cenários para preço do ATR e produtividade da cana (safra 2015/2016)

\begin{tabular}{c|cccc}
\hline \multirow{2}{*}{$\begin{array}{c}\text { Margem } \\
\text { CT }\end{array}$} & \multicolumn{4}{|c}{ Preço ATR $\left(\mathrm{R} \$ \mathrm{~kg}^{-1}\right.$ ATR $)$} \\
\cline { 2 - 5 } Produtividade & 0,5605 & Base & Otimista & Atual \\
\hline t ha $^{-1}$ & & 0,5900 & 0,6195 & 0,5965 \\
57 & $-41,72$ & $-36,58$ & $-31,50$ & $-39,13$ \\
60 & $-39,60$ & $-34,27$ & $-29,00$ & $-36,92$ \\
63 & $-37,55$ & $-32,03$ & $-26,57$ & $-34,78$ \\
\hline
\end{tabular}

Fonte: PECEGE/CNA, 2015

Tabela 6. Análise de sensibilidade para a rentabilidade agrícola (margem COT): cenários para preço de ATR e produtividade da cana (safra 2015/16)

\begin{tabular}{c|cccc}
\hline \multirow{2}{*}{$\begin{array}{c}\text { Margem } \\
\text { COT }\end{array}$} & \multicolumn{4}{|c}{ Preço ATR $\left(\mathrm{R} \$ \mathrm{~kg}^{-1}\right.$ ATR $)$} \\
\cline { 2 - 5 } Produtividade & 0,5605 & Base & Otimista & Atual \\
\hline t ha $^{-1}$ & \multicolumn{4}{|c}{$\%$} \\
57 & $-31,50$ & $-25,24$ & $-19,02$ & $-28,5800$ \\
60 & $-29,21$ & $-22,73$ & $-16,30$ & $-26,19$ \\
63 & $-26,99$ & $-20,31$ & $-13,67$ & $-23,88$ \\
\hline
\end{tabular}

Fonte: PECEGE/CNA, 2015

Ao contrário do que vinha acontecendo nas últimas quatro safras, projeta-se uma ligeira recuperação do preço-base ATR, em termos reais. Não obstante, para o cenáriobase destaca-se o custo operacional total superior ao preço - cerca de $R \$ 12,90 \mathrm{~kg}^{-1}$ ATR. Estima-se que produtores e fornecedores de cana-de-açúcar atingirão rentabilidade positiva sobre o custo total - CT apenas para produtividades superiores a 100 t ha-1 $^{-1}$. Por outro lado, se for mantida a produtividade base, a qualidade da cana deve ser superior à 179,7 kg ATR t-1 para o CT e, para o COT, superior a 152,9 kg ATR t ${ }^{-1}$, para que seja mantida a margem positiva.

As Tabelas 7 e 8 apresentam as expectativas de rentabilidade do açúcar VHP ${ }^{7}$ seguida pelo branco. Espera-se aumento de preços para ambos os produtos, no entanto, o único produto que se mostra rentável é o açúcar branco, em apenas um dos cenários-base e em todos os otimistas, obtendo margem positiva para produtividades

\footnotetext{
${ }^{7}$ Nos cenários, os preços dos produtos consideram variações em conjunto, ou seja, o cálculo da rentabilidade de cada cenário do açúcar VHP considera variação proporcional nos preços dos demais produtos e no preço do ATR em relação às premissas adotadas no modelo.
} 
acima de 60,9 t ha ${ }^{-1}$. O açúcar VHP, por outro lado, não obteve margem positiva em nenhuma das 12 possibilidades projetadas, alcançando rentabilidades apenas para valores de produtividade acima de 75,5 t ha ${ }^{-1}$, aos níveis de preços médios estimados para a safra 2015/16.

Tabela 7. Análise de sensibilidade para a rentabilidade (margem CT) na safra 2015/16: cenários para produtividade agrícola da cana e preços de açúcar VHP

\begin{tabular}{c|cccc}
\hline \multirow{2}{*}{$\begin{array}{c}\text { Margem } \\
\text { CT }\end{array}$} & \multicolumn{4}{|c}{ Açúcar VHP $\left({\left.\mathrm{R} \$ \mathrm{t}^{-1}\right)}\right.$} \\
\cline { 2 - 5 } Produtividade & Pessimista & Base & Otimista & Atual \\
\hline t ha $^{-1}$ & 950 & 1000 & 1050 & 850 \\
57 & $-15,98$ & $-12,66$ & $-9,42$ & $-25,94$ \\
60 & $-13,70$ & $-10,31$ & $-7,00$ & $-23,95$ \\
63 & $-11,54$ & $-8,07$ & $-4,70$ & $-22,06$ \\
\cline { 2 - 5 }
\end{tabular}

Fonte: PECEGE/CNA, 2015

Tabela 8. Análise de sensibilidade para a rentabilidade (margem CT) na safra 2015/16: cenários para preço e produtividade de açúcar branco

\begin{tabular}{c|cccc}
\hline \multirow{2}{*}{$\begin{array}{c}\text { Margem } \\
\text { CT }\end{array}$} & \multicolumn{4}{|c}{ Açúcar Branco $\left({\left.\mathrm{R} \$ \mathrm{t}^{-1}\right)}\right.$} \\
\cline { 2 - 5 } & Pessimista & Base & Otimista & Atual \\
\hline Produtividade & 1102 & 1160 & 1218 & 1206 \\
\hline t ha $^{-1}$ & & & $\%$ \\
57 & $-7,08$ & $-3,36$ & 0,27 & 0,23 \\
60 & $-4,46$ & $-0,66$ & 3,06 & 3,03 \\
63 & $-1,96$ & 1,93 & 5,72 & 5,70 \\
\hline
\end{tabular}

Fonte: PECEGE/CNA, 2015

O açúcar VHP, principal produto da região, cedeu ligeiro espaço para o etanol, devido às condições macro e microeconômicas já descritas, de baixo preço internacional do açúcar VHP e cenário nacional favorável à produção de etanol, com o reestabelecimento parcial da CIDE incidente sobre a gasolina e a elevação da porcentagem de etanol anidro na gasolina.

Ainda assim, o etanol não se mostra mais rentável em comparação com os outros produtos, conforme evidencia a rentabilidade do etanol anidro na Tabela 9. 
Tabela 9. Análise de sensibilidade para a rentabilidade (margem CT) na safra 2015/16: cenários para preço e produtividade do etanol anidro

\begin{tabular}{c|cccc}
\hline \multirow{2}{*}{$\begin{array}{c}\text { Margem } \\
\text { CT }\end{array}$} & \multicolumn{4}{|c}{ Etanol Anidro $\left(\mathrm{R} \$ \mathrm{~m}^{-3}\right)$} \\
\cline { 2 - 5 } Produtividade & 1598 & Base & Otimista & Atual \\
\hline t ha $^{-1}$ & & \multicolumn{3}{|c}{$\%$} \\
57 & $-15,98$ & $-12,66$ & $-9,43$ & $-12,73$ \\
60 & $-13,70$ & $-10,31$ & $-7,01$ & $-10,35$ \\
63 & $-11,54$ & $-8,08$ & $-4,71$ & $-8,09$ \\
\hline
\end{tabular}

Fonte: PECEGE/CNA, 2015

A produção sucroenergética, em mais uma safra projeta-se como uma atividade econômica de baixa atratividade em relação a investimentos em outros setores da economia brasileira, mesmo em cenários otimistas. Ademais, expectativa de aumento do custo total de produção de cana-de-açúcar aponta para maiores incentivos à aquisição de cana de terceiros. Pelo lado positivo, é esperado que as usinas com processos mais otimizados tenham possibilidade de obter equilíbrio econômico.

A partir das projeções dos cenários elaboradas, torna-se possível inferir que a safra atual no Nordeste aponta para uma menor produtividade e menor qualidade da matéria-prima. Excetuando o açúcar branco, há baixa rentabilidade para a maior parte dos produtos, apesar da perspectiva de aumento nominal dos preços. A Figura 1 mostra a relação preço-custo, em termos reais, entre os produtos ao longo das últimas 6 safras. A linha tracejada destaca o ponto em que o custo é igual ao preço.



Figura 1. Médias e projeção para a relação preço-custo dos principais produtos derivados da cana entre 2007/08 e 2015/16, em valores reais com base em agosto/2015 Fonte: PECEGE/CNA, 2015 
O açúcar apresenta relação preço-custo favorável historicamente. Por outro lado, a média das últimas 3 safras mostra que este indicador está em queda. Ainda que, para o açúcar branco, o custo seja igual ao preço, na safra 2015/16, ambos os produtos estão distantes de suas médias históricas. Para o açúcar VHP, conforme já mencionado, isso se deve à queda de preços no mercado internacional. Por outro lado, pode-se observar uma melhora dessa relação para o etanol anidro e para o etanol hidratado, com perspectiva de se manter melhorando no curto prazo.

Com a perspectiva de cenários pouco rentáveis, a solução para obter margens operacionais no setor passa pela necessidade de elevar a produtividade dos canaviais. Devido à menor disponibilidade de recursos financeiros ao longo das últimas safras espera-se diminuição da área colhida, dos gastos com tratos de cana e da sua qualidade.

Apesar de a produtividade do canavial ter se recuperado desde as quebras de produção nas safras 2009/10 e 2012/13, a oferta de ATR por hectare dos canaviais está tão reduzida quanto nas safras em que houve quebra de produtividade (devido ao reduzido nível de ATR da cana). A Figura 2 destaca a produtividade agrícola e a quantidade de ATR na cana própria desde a safra 2007/08 até o projetado para a safra 2015/16.

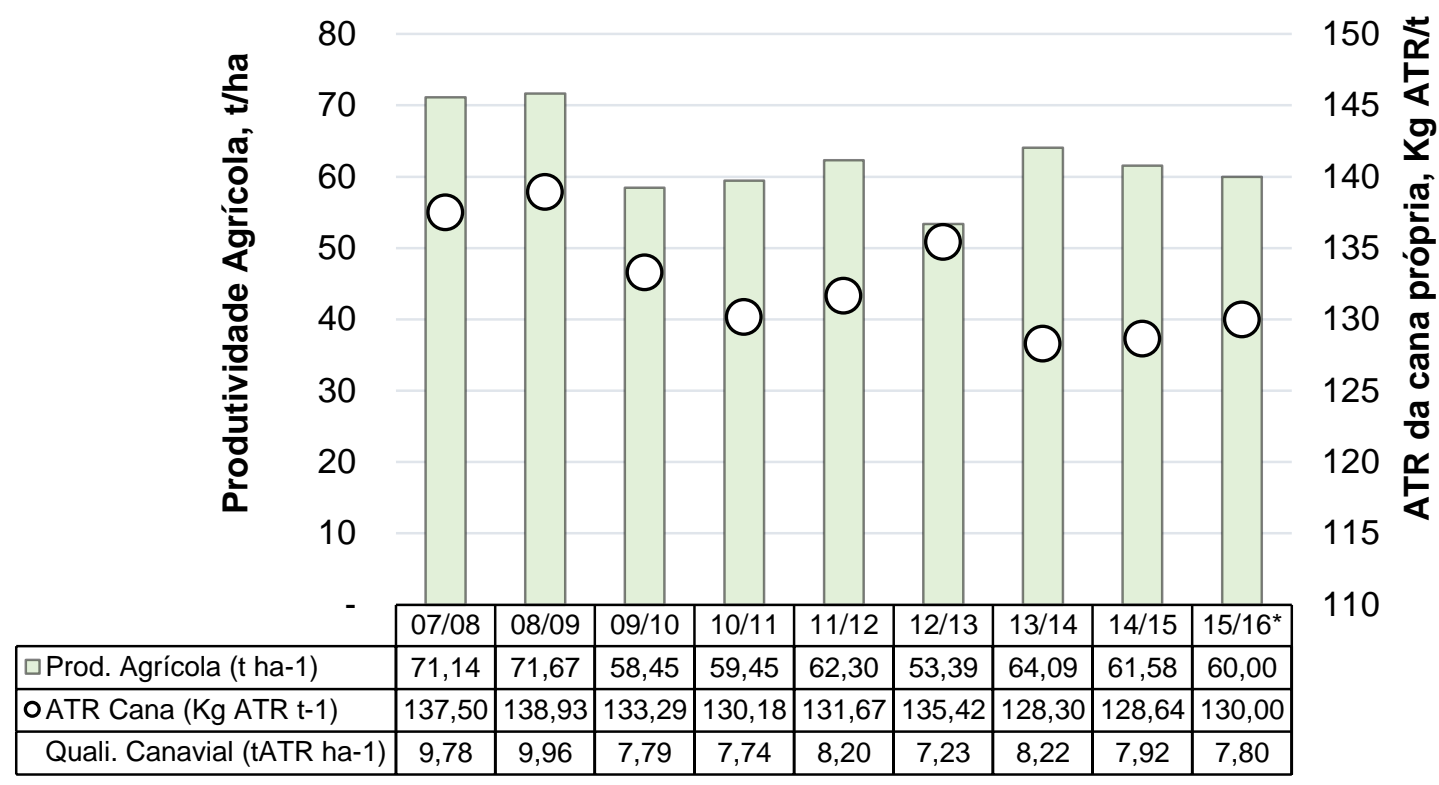

Figura 2. Evolução da produtividade agrícola e do ATR da cana própria - safra 2007/08 a $2015 / 16$

Fonte: PECEGE/CNA, 2015

Nota: * Projeção 
Para que as usinas obtenham rentabilidade econômica é necessário que otimizem seus processos produtivos. O crescente endividamento e o aumento dos custos de produção, contudo, impõem grandes desafios às unidades produtoras que precisam investir para reverter a deterioração da qualidade dos canaviais. Pelo lado positivo, espera-se que o preço pago pelos produtos aumente no curto prazo. O câmbio atenuará a redução dos preços internacionais do açúcar e o aumento do preço da gasolina no mercado doméstico darão novo fôlego e competitividade ao etanol.

\section{Considerações Finais}

A baixa rentabilidade e os custos mais altos projetados para o setor sucroenergético apontam para as dificuldades enfrentadas pelas usinas. Assim, ainda que o mix continue sendo mais direcionado para a produção de açúcar, a expectativa para a safra 2015/2016 é de um aumento na participação da produção de etanol. Os aspectos macroeconômicos que justificam essa decisão são principalmente a retração do crédito no mercado doméstico e o encarecimento dos insumos pressionados pela desvalorização cambial e pela necessidade de geração de caixa pelas usinas.

A queda em dólar no preço internacional do açúcar se sobrepõe à desvalorização do real ante a moeda americana e acomete a comercialização deste produto. Por fim, o reestabelecimento parcial da CIDE incidente sobre a gasolina e a elevação da porcentagem de etanol anidro na gasolina, ajuda a compor um cenário que favorece o investimento em etanol.

Além da elevação na produção de etanol, a redução de custos através da otimização dos canaviais e do processo industrial são necessárias para enfrentar o atual momento produtivo do setor e impõe grande desafio. Assim, as usinas que puderem investir devem se manter no setor, enquanto as menos capitalizadas terão dificuldades correm o risco de não reverter o ciclo negativo.

\section{Referências}

Programa de Educação Continuada em Economia e Gestão de Empresas [PECEGE]. 2014. Custos de produção de cana-de-açúcar, açúcar, etanol e bioeletricidade no Brasil: Fechamento da safra 2013/2014. Piracicaba: Universidade de São Paulo, Escola Superior de Agricultura "Luiz de Queiroz", Programa de Educação Continuada em Economia e Gestão de Empresas/Departamento de Economia, Administração e Sociologia. 2014. 53 p. Relatório apresentado à Confederação da Agricultura e Pecuária do Brasil - CNA. 